\title{
ON MINIMAL BLOCKS $\left({ }^{1,2}\right)$
}

\author{
BY \\ MICHAEL D. PLUMMER
}

1. Introduction. A connected graph $G$ is a block if there is no point $v$ in $G$ such that $G-v$ is disconnected. A block $G$ will be called block-line-critical (b.l.c.) if, for every line $x$ in $G, G-x$ is not a block. Such graphs occur repeatedly in the study of blocks-for example, when a proof by induction on the number of lines of a block is being attempted.

Clearly, a cycle of any length is a b.l.c. graph. There are, however, b.l.c. graphs with a much more complex structure. In this paper several structural characterizations of b.l.c. graphs are obtained as well as a number of additional properties of such graphs.

2. Additional terminology. For the sake of completeness, we introduce the following additional definitions. A graph $G$ is a finite nonempty set $V(G)$ of points together with a collection $E(G)$ of lines each of which is an unordered pair of points. If $x$ is the line containing the points $u$ and $v$, we write $x=u v$ and say that $u$ and $v$ are adjacent, $x$ joins $u$ and $v$, and that $x$ is incident with points $u$ and $v$. Two lines $x$ and $y$ which have a common point are also said to be adjacent. The complete graph on $p$ points, $K_{p}$, is that graph with $p$ points in which every two points are adjacent. A subgraph of $G$ is a graph all of whose points and lines are also in $G$. The subgraph of a graph $G$ generated by a set of lines $X$ is that graph $H$ whose set of lines is $X$ and whose points are those points of $G$ incident with a line of $X$.

A path $P$ is an alternating sequence of distinct points and lines, beginning and ending with points (said to be joined by $P$ ) such that each line is incident with the points before and after it. The first and last points in this sequence are called the endpoints of $P$, and all other points are termed intermediate. We shall have occasion to refer to a path $P$ by its sequence of points; e.g., $P=\left[u_{1}, u_{2}, \ldots, u_{n}\right]$. If $P=$ $\left[u_{1}, u_{2}, \ldots, u_{m}\right]$ and $Q=\left[v_{1}, v_{2}, \ldots, v_{n}\right]$ are two paths where the intermediate points of $P$ are all distinct from the intermediate points of $Q, u_{m}=v_{1}$, and either $v_{n}=u_{1}$ or $v_{n}$ is distinct from every point of $P$, we define a new path $P+Q$, the sum of $P$ and $Q$, to be that path with point sequence $\left[u_{1}, u_{2}, \ldots, u_{m}=v_{1}, v_{2}, \ldots, v_{n}\right]$. A path of length $\geqq 2$ together with a line joining the first and last points is called a cycle. A path or a

\footnotetext{
Received by the editors November 7, 1966 and, in revised form, January 6, 1967 and June 6, 1967.

(1) Work supported in part by the National Science Foundation under Grant NSF GP-6558.

(2) The author wishes to thank Professor L. W. Beineke and Dr. B. Rothschild for their thoughtful suggestions leading to several shorter theorem proofs.
} 
cycle is odd (even) if the number of lines in it is odd (even). As for paths, we shall have need to designate a cycle by the sequence of its points; e.g., $C=\left[u_{1}, u_{2}, \ldots\right.$ $u_{n}, u_{1}$ ]. If $C$ is a cycle in a graph $G$ and $x$ is a line of $G$ which is not in $C$, but which joins two points of $C$, then $x$ is called a diagonal of $C$. More generally, a line $x$ is a diagonal of a graph $G$ if it is a chord of some cycle in $G$.

The graph $G$ is connected if every two points are joined by a path. If $x$ is a line of $G, G-x$ will denote the graph obtained from $G$ by deleting $x$. Similarly, $G-v$ will denote the graph obtained from $G$ by deleting the point $v$ and all lines incident with $v$. More generally, if $N$ is any set of points or lines in $G, G-N$ will denote the graph obtained by deleting each element of the set $N$. A point $v$ is a cutpoint of the connected graph $G$ if $G-v$ is not connected, and the set $N$ is called a separating (or disconnecting) set for the connected graph $G$ if $G-N$ is disconnected. A line $x$ is called a bridge in a connected graph $G$ if $G-x$ is disconnected. The cardinality of any minimum separating set of lines in $G$ is called the line-connectivity of $G$ and is denoted $\lambda(G)$. If $v$ is any point of a graph $G$, a branch of $G$ at $v$ is a maximal connected subgraph of $G$ not having $v$ as a cutpoint. A connected graph is a block if it has no cutpoints. A line $x$ in a block $B$ is said to be critical with respect to $B$ if $B-x$ is not a block. A connected graph containing no cycles is a tree.

The degree of a point $v, d(v)$, is the number of lines incident with it. A set of points is said to be independent if no two of its members are adjacent. Let $|M|$ denote the number of elements in a set $M$. If $M$ is a maximum independent set of points in $G,|M|$ is called the point independence number of $G$ and is denoted by $\beta_{0}(G)$. A set of points $M$ is said to cover a graph $G$ if every line of $G$ has at least one point in $M$. If $M$ is a point cover for $G$ with a minimum number of elements, then $|M|$ is called the point covering number of $G$ and is denoted by $\alpha_{0}(G)$. Finally, a graph $G$ is bipartite if $V(G)$ can be partitioned into two nonempty subsets $V_{1}$ and $V_{2}$ so that every line of $G$ joins a point of $V_{1}$ and a point of $V_{2}$. Let $m$ and $n$ be the number of points in $V_{1}$ and $V_{2}$, respectively. The complete bipartite graph $K(m, n)$ is the bipartite graph in which every point of $V_{1}$ is adjacent to every point of $V_{2}$.

3. Characterization of block-line-critical graphs. The following theorem immediately yields a first characterization of b.l.c. graphs.

THEOREM 1. Let $G$ be a block and $x$ a line of $G$. Then $G-x$ is a block if and only if $x$ is a diagonal in $G$.

Proof. Clearly, if $x$ is a diagonal of $G$, then $G-x$ is still a block. On the other hand, let $x=u v$ be a line in $G$ and assume that $G-x$ is a block. Then in $G-x$ there must be a cycle containing $u$ and $v$ and $x$ is a diagonal of this cycle.

COROLlaRY 1a. The following three conditions are equivalent for any graph $G$ :

(1) $G$ is block-line-critical,

(2) $G$ is a block containing no diagonals, and

(3) $G$ is a block in which no point is adjacent to three points of the same cycle. 
Corollary 1b. If $G$ is a b.l.c. graph and $G \neq K_{3}$, then $G$ contains no triangles.

Proof. Suppose $G$ has a triangle with points $u, v$, and $w$. At least one of these, say $u$, is incident with a line $x$ not in the triangle. But then there must be a cycle containing lines $x$ and $v w$, and clearly either $u v$ or $v w$ is a diagonal of this cycle.

A cycle is, of course, a b.l.c. graph in which every point has degree two. We next proceed to show that any b.l.c. graph must contain points of degree two and that such points have an important bearing upon the structure of the b.l.c. graph.

THEOREM 2. Let $x=u v$ be any line of $a$ b.l.c. graph $G$ and let $w$ be any cutpoint of $G-x$. Then

(1) $w$ is on every cycle containing $x$, and

(2) every path containing $x$ and $w$ has an intermediate point of degree two in $G$.

Proof. For (1), it is sufficient to show that $u$ and $v$ are in different blocks of $G-x$. But if $u$ and $v$ were in the same block of $G-x$, they would lie on a cycle in this block and such a cycle would have $x$ as a diagonal in $G$, thus contradicting Corollary la.

We proceed to prove (2). Let $B$ be the block of $G-x$ containing $v$. Let $w$ be the cutpoint of $G$-x contained in $B$. Let $P=\left[u, v, v_{1}, v_{2}, \ldots, v_{n}, w\right]$ be a path in $G$ (cf. Figure 1).

We assume that the degree of each point $v=v_{0}, v_{1}, v_{2}, \ldots, v_{n}, v_{n+1}=w$ is at least three in $G$. Form a new graph $A$ from $B$ by deleting the lines of the path $P$. In $A$, each $v_{i}$ must be joined to another $v_{j}(i \neq j)$ by a path $Q$ which has no intermediate points in $P$. Moreover, $Q$ cannot join $v_{i}$ and $v_{i+1}$ since the line $v_{i} v_{i+1}$ would be a diagonal of the cycle determined by $Q, P-v_{i} v_{i+1}$, and any path of $G-x$ joining $w$ and $u$ and not containing $v$. In particular, $v_{0}$ is joined by a path in $A$ to $v_{k}, k>1$. Let

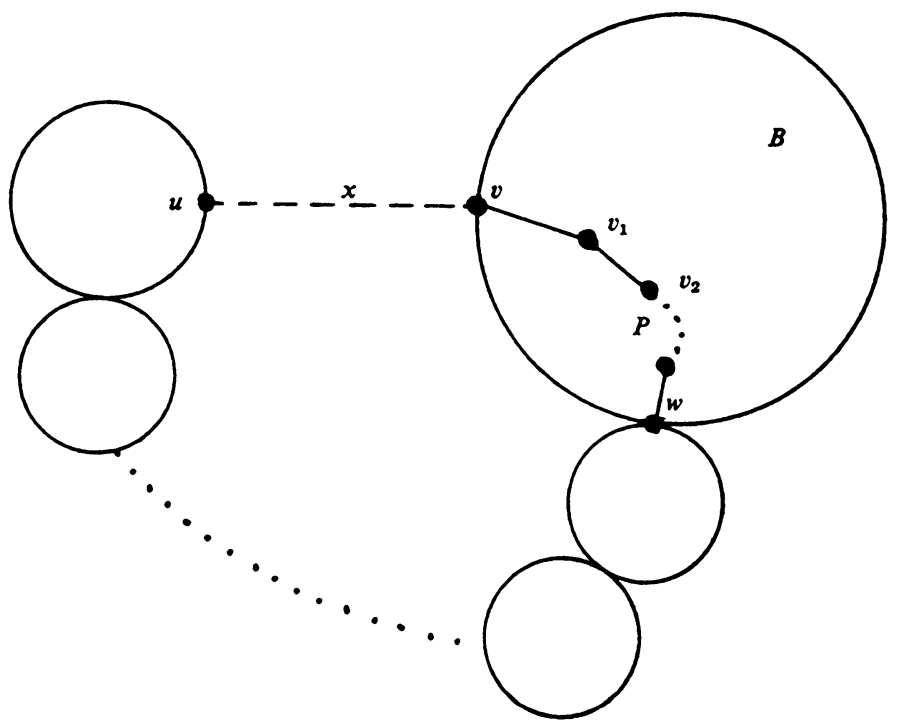

FIGURE 1 
$\alpha$ denote the greatest subscript $i$ such that $i$ is joined by a path $Q$ in $A$ to some $v_{m}$, $m>i$. Clearly, $0 \leqq \alpha \leqq n$. But then $v_{\alpha+1}$ must be joined by a path $R$ in $A$ to some $v_{\beta}$, $\beta<\alpha$. Furthermore, $Q$ and $R$ are disjoint since otherwise there would be a path from $v_{\alpha}$ to $v_{\alpha+1}$ in $A$. Thus one can form a cycle from $Q, R$, and $P-v_{\alpha} v_{\alpha+1}$ (cf. Figure 2) which has $v_{\alpha} v_{\alpha+1}$ as a diagonal, contradicting Corollary la and completing the proof of the theorem.

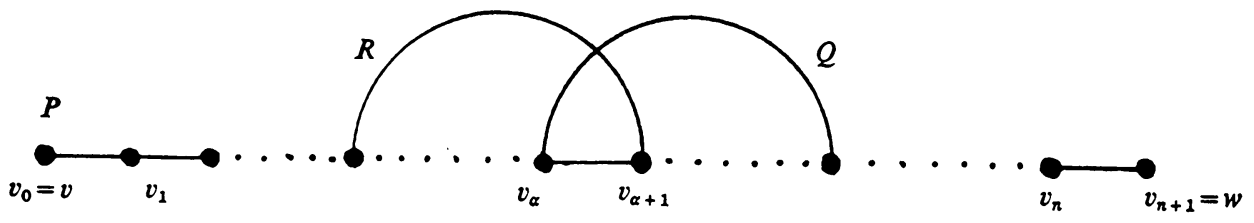

FIGURE 2

The following corollary is immediate.

COROLlaRY 2a. If $G$ is a b.l.c. graph $\neq K_{3}$, and $C$ is any cycle in $G$, then $C$ contains at least one pair of independent points whose degree in $G$ is two.

According to Theorem 2, every cycle in a b.l.c. graph is separated by points of degree two. We next proceed to show that the graph as a whole is separated by these points.

Let $G^{\prime}$ be the subgraph of $G$ generated by all points of degree greater than two and let $T_{1}, T_{2}, \ldots, T_{s}$ denote the components of $G^{\prime}$. By Theorem 2, $G^{\prime}$ contains no cycles. Hence each $T_{i}$ is a tree. Denote by $S$ the set of points in $G$ of degree two. A path $P$ will be called a $S$-path if each of its intermediate points is of degree two in $G$.

THEOREM 3. If $G$ is a b.l.c. graph and if $S$ and $T_{i}$ are as defined above, then there is no $S$-path joining two points of the same tree $T_{i}$.

Proof. Suppose the conclusion to be false; i.e., suppose that $\left[v_{0}, v_{1}, \ldots, v_{n}\right]$ is an $S$-path with $v_{0}$ and $v_{n}$ in the same tree $T$ of $G-S$. Clearly $v_{n}$ is a cutpoint of $G-v_{0} v_{1}$. But the path of $T$ joining $v_{0}$ and $v_{n}$ has no points of degree 2 , contradicting Theorem 2.

We then have the following result.

Corollary 3a. A b.l.c. graph $G$ is separated by its points of degree 2.

Proof. Suppose $G-S$ is connected. Then by Theorem 2 , it contains no cycles and thus must be a tree. But then any $S$-path must join two points of $G-S$, contradicting Theorem 3.

To summarize then, a b.l.c. graph consists of at least two mutually point-disjoint trees $T_{1}, T_{2}, \ldots, T_{s}$ and a collection of paths joining pairs of $T_{i}$ 's, where the degree of all intermediate points of such a path is two. 
Next it is shown that the array of $S$-paths in a b.l.c. graph is not completely arbitrary, but rather their location must be such so as to avoid the formation of cycles of a certain type. More specifically, we have the next theorem.

THEOREM 4. Let $G$ be a b.l.c. graph and let $T_{1}, T_{2}, \ldots, T_{s}(s \geqq 2)$ be the component trees of $G$-S. Let $C$ be any cycle in $G$. Then for each j, either $C \cap T_{j}=\varnothing$, or $C \cap T_{j}$ is connected.

Proof. Suppose there is a cycle $C$ in $G$ and a component tree $T_{k}$ of $G-S$ such that $C \cap T_{k}$ is disconnected. Let $D_{1}, \ldots, D_{r}$ be the components of $C \cap T_{k}(r>1)$. Now given $i$ and $j$, there is a path $P_{i j}$ in $T_{k}$ joining a point of $D_{i}$ and a point of $D_{j}$. Suppose $P_{0}$ is a path of minimum length joining two components of $C \cap T_{k}$, where the minimum is taken over all cycles $C$ and all trees $T_{k}, k=1, \ldots, s$, such that $C \cap T_{k}$ is disconnected. For the sake of argument, suppose $P_{0}$ joins two components of the intersection of the cycle $C_{0}$ and the tree $T_{1}$.

Observe first that since $G$ contains no diagonal, $P_{0}$ consists of at least two lines. Let $P_{0}=\left[u=u_{0}, u_{1}, \ldots, u_{t}=v\right]$, where $u$ and $v$ are in $V\left(C_{0}\right)$. Now since $d\left(u_{1}\right)>2$, there is a line $y=u_{1} w_{1}$ where $u_{0} \neq w_{1} \neq u_{2}$. If $w_{1} \in V\left(C_{0}\right)-\{v\}$, then one may obtain a cycle with $x-u_{0} u_{1}$ as a diagonal contrary to Theorem 1 . Hence $w_{1} \notin V\left(C_{0}\right)-\{v\}$. Also, if $w_{1} \in V\left(P_{0}\right)$, then the length of $P_{0}$ would not be minimal, hence $w_{1} \notin V\left(P_{0}\right)$.

Since $G$ is a block, there is a cycle $C_{1}$ containing $x$ and $y$. Let $B_{1}$ be the branch of $C_{1}-x-y$ traversed by starting at $w_{1}$ and terminating upon the first encounter with a point $p_{0}$ of $V\left(C_{0}\right) \cup V\left(P_{0}\right)$. Once again a diagonal may be obtained if $p_{0} \in V\left(C_{0}\right)$ $-\{v\}$. Hence we may assume that $p_{0} \in V\left(P_{0}\right)-\{u\}$ (cf. Figure 3).

Now not all lines of $B_{1}+y$ are in $T_{1}$, for if they were, $B_{1}+y+\left[u_{1}, u_{2}, \ldots, p_{0}\right]$ would be a cycle in $T_{1}$, contrary to the assumption that $T_{1}$ is a tree. Let $C_{L}$ be one of the two paths in $C_{0}$ determined by the points $u$ and $v$. Then $E\left(C_{L}\right) \notin E\left(T_{1}\right)$.

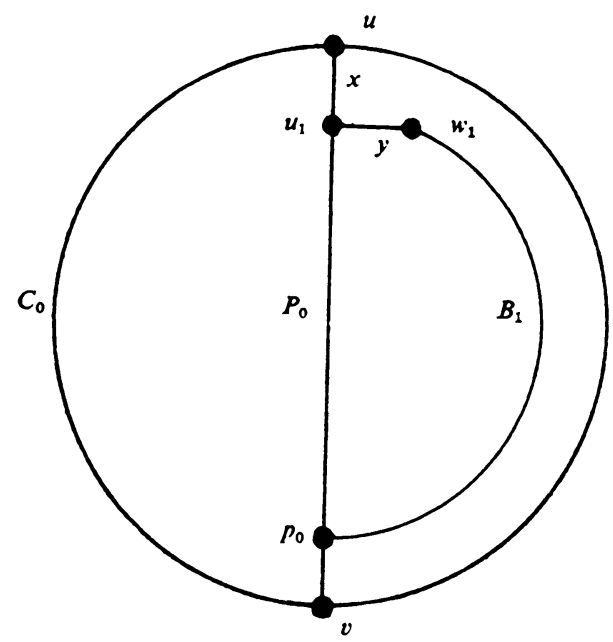

FIGURE 3 
Hence, if $P_{0}^{\prime}$ is the subpath of $P_{0}$ with endpoints $p_{0}$ and $v$, then $C^{\prime}=C_{L}+x+y+B_{1}$ $+P_{0}^{\prime}$ is a cycle and $C^{\prime} \cap T_{1}$ is disconnected. But then $P_{0}^{\prime \prime}=\left[u_{1}, u_{2}, \ldots, p_{0}\right]$ is a path in $T_{1}$ joining two components of $C^{\prime} \cap T_{1}$ and the length of $P_{0}^{\prime \prime}$ is less than that of $P_{0}$. This contradicts the minimality of the length of $P_{0}$ and completes the proof of the theorem.

The following construction will prove useful to us later. If $G$ is a b.l.c. graph, we define its tree-condensation graph $G^{*}$ as follows: the points of $G^{*}$ are the component trees of $G-S, T_{1}, T_{2}, \ldots, T_{s}$. Two points $T_{i}$ and $T_{j}$ are adjacent in $G^{*}$ if and only if there is some $S$-path joining them in $G$. We may apply Theorem 4 to obtain the following result.

Corollary 4a. If $G$ is a b.l.c. graph, then $G^{*}$ is a block.

Proof. Suppose $T_{i}$ is a cutpoint in $G^{*}$. Let $T_{j}$ and $T_{k}$ be two points of $G^{*}$ adjacent to $T_{i}$, but which lie in different components of $G^{*}-T_{i}$. Let $P_{i j}$ and $P_{i k}$ be $S$-paths joining $T_{i}$ and $T_{j}$ and $T_{i}$ and $T_{k}$ respectively. Let $x$ be a line of $P_{i j}$ and $y$, a line of $P_{i k}$. Now $x$ and $y$ lie on a cycle $C$ in $G$. But then $C \cap T_{j} \neq \varnothing$ and is disconnected, contrary to Theorem 4 . Hence $G^{*}$ has no cutpoints and the corollary is proved.

We may now use the properties of b.l.c. graphs derived in Theorems 2,3 , and 4 to characterize this family of graphs.

THEOREM 5. Let $G$ be a block. Then $G$ is a b.l.c. graph if and only if:

(1) $G$ is a cycle, or

(2) if $S$ denotes the set of points of degree two in $G$, then there are at least two components $T_{j}$ in G-S, each component of G-S is a tree, and if $C$ is any cycle in $G$, for each $j$ either $C \cap T_{j}=\varnothing$ or $C \cap T_{j}$ is connected.

Proof. If $G$ is a b.l.c. graph, then (1) or (2) holds by Theorems 2, 3, and 4.

On the other hand, if $G$ is a cycle, it is clearly b.l.c.. So suppose $G-S$ has components $T_{1}, \ldots, T_{s}, s \geqq 2$, where each $T_{j}$ is a tree and each has the cycle intersection property stated in the hypotheses. Let $x=u v$ be any line of $G$. If either $d(u)=2$ or $d(v)=2$, clearly $G-x$ is not a block. Otherwise, $x$ is a line in $T$, for some $j$. Now let the components of $T_{j}-x$ be $T_{j}^{\prime}$ and $T_{j}^{\prime \prime}$ where $u \in V\left(T_{j}^{\prime}\right)$ and $v \in V\left(T_{j}^{\prime \prime}\right)$. Suppose $G-x$ is a block. Then $u$ and $v$ lie on a cycle $C$ in $G$-x. Hence $C^{\prime}=C \cap T_{j}^{\prime} \neq \varnothing$ and $C^{\prime \prime}=C \cap T_{j}^{\prime \prime} \neq \varnothing$. But $T_{j}^{\prime} \cap T_{j}^{\prime \prime}=\varnothing$, hence $C^{\prime} \cap C^{\prime \prime}=\varnothing$ and $C \cap T_{j}$ is disconnected, contrary to assumption. This completes the proof of the theorem.

4. Further properties of block-line-critical graphs. We next turn our attention to some additional properties of b.l.c. graphs. The chromatic number of a graph $G$, $\chi(G)$, is the minimum number of colors needed to color the points of $G$ so that no two adjacent points have the same color. Clearly, any bipartite graph has chromatic number two. This observation, together with the next theorem, completely determines $\chi(G)$ for any b.l.c. graph $G$.

THEOREM 6. If $G$ is a b.l.c. graph, and $G$ contains an odd cycle, then $\chi(G)=3$. 
Proof. This is clear from our previously gained knowledge of the structure of any b.l.c. graph $G$. For if $G$ is a cycle, the theorem is immediate. Otherwise, let the component trees of $G-S$ be $T_{1}, T_{2}, \ldots, T_{s}, s \geqq 2$. Since these trees are bipartite, each can be colored with two colors. Moreover, if $P$ is any $S$-path joining two trees $T_{i}$ and $T_{j}$, then it requires at most three colors to color the points of $P$, if the endpoints are already colored. On the other hand, since $G$ contains an odd cycle, at least three colors are required to color the points of $G$. Hence $\chi(G)=3$ and the Theorem is proved.

We may combine the result of Theorem 6 with a well-known inequality involving the point independence number of a b.l.c. graph $G, \beta_{0}(G)$.

COROLlaRY 6a. If $G$ is a b.l.c. graph, then $\beta_{0}(G) \geqq|V(G)| / 3$.

Proof. It is well known (cf. Ore [2]) that $\beta_{0}(G) \chi(G) \geqq|V(G)|$, for any graph. This, combined with the result of Theorem 6 , yields the desired inequality.

One may use a well-known result of Gallai [1], which says that $\alpha_{0}(G)+\beta_{0}(G)$ $=|V(G)|$, to state the conclusion of Corollary 6 a as: $\alpha_{0}(G) \leqq 2|V(G)| / 3$.

Since any b.l.c. graph $G$ must contain a point of degree two, the line-connectivity of $G, \lambda(G)$, must be equal to two. Let $G_{0}$ denote the subgraph of $G$ generated by all lines having at least one endpoint of degree two in $G$. We now proceed to investigate the possible locations of minimum disconnecting sets of two lines. There are, a priori, four possible types:

(A) both lines in $G_{0}$,

(B) one line in $G_{0}$, the other in $T_{i}$ for some $i$,

(C) one line in $T_{i}$, the other in $T_{j}$, for $i \neq j$, or

(D) both lines in $T_{i}$, for some $i$.

If $x$ and $y$ are lines on the same $S$-path in $G_{0}$, then $\{x, y\}$ is a set of type (A) and every b.l.c. graph possesses minimum disconnecting sets of this kind. There may also be sets $\{x, y\}$ of type (A), where $x$ is on an $S$-path joining $T_{i}$ and $T_{j}, y$ is on an $S$-path joining $T_{k}$ and $T_{m}$, and where $T_{i}, T_{j}, T_{k}$, and $T_{m}$ are all distinct. The next theorem shows that these are the only two possibilities for sets of type (A).

THEOREM 7. If $\{x, y\}$ is a minimum disconnecting set of type (A) for a b.l.c. graph $G$ which is not a cycle, if $x$ lies on an $S$-path joining $T_{i}$ and $T_{j}$ and if $y$ lies on an $S$-path $Q$ joining $T_{k}$ and $T_{m}$, then either $i, j, k$, and $m$ are all distinct, or $i=k$ and $j=m$ (or $i=m$ and $j=k$ ) and then $P$ and $Q$ are identical.

Proof. By Theorem $4, i \neq j$ and $k \neq m$. Suppose $i=k$. Then if $j \neq m, T_{i}$ is a cutpoint of $G^{*}$, contradicting Corollary 4 a. Thus $j=m$. Then, if $P$ and $Q$ are not identical, the line of $G^{*}$ containing $P$ and $Q$ is a bridge in $G^{*}$. But then $G^{*}=K_{2}$, or else Corollary $4 \mathrm{a}$ is again contradicted. But if $G^{*}=K_{2}$, since $\{x, y\}$ is a disconnecting set, there are no other $S$-paths joining $T_{i}$ and $T_{j}$ and hence no other $S$-paths in $G$. But then since $G$ is a block, it follows that $T_{i}$ and $T_{j}$ each have but one point which 
therefore must be of degree two, contradicting the definitions of $T_{i}$ and $T_{j}$. The proof is complete.

We have the following restrictions on sets of type (B).

THEOREM 8. If $G$ is a b.l.c. graph, $\{x, y\}$ is a set of type (B), $x$ is a line in $T_{i}$, and $y$ lies on an $S$-path $P$ joining $T_{j}$ and $T_{k}$, then $i \neq j$ and $i \neq k$.

Proof. Suppose $\{x, y\}, T_{i}, T_{j}, T_{k}$, and $P$ are as given in the hypothesis. Assume $i=j$. Now let $T_{i}^{\prime}$ and $T_{i}^{\prime \prime}$ be the components of $T_{i}$ - $x$. Let $x=u v$, where $u \in V\left(T_{i}^{\prime}\right)$ and $v \in V\left(T_{i}^{\prime \prime}\right)$. Let $z$ be a line of $G_{0}$ with exactly one endpoint in $T_{i}^{\prime}, z \neq x$, and suppose $z$ is not on the path $P$. (Such a line must exist by definition of $T_{i}^{\prime}$.) Now $y$ and $z$ must lie on a cycle $C$, which must necessarily contain $x$. But then $C$ is a cycle in $G$, $C \cap T_{i} \neq \varnothing$, and $C \cap T_{i}$ is disconnected, contradicting Theorem 4. Hence $i \neq j$, and similarly $i \neq k$, completing the proof.

Finally, we show that type (D) sets do not exist.

THEOREM 9. If $G$ is a b.l.c. graph, $\{x, y\}$ is a minimum disconnecting set, $x$ is in $T_{i}$, and $y$ is in $T_{j}$, then $i \neq j$.

Proof. Suppose $x$ and $y$, as given in the hypothesis, both lie in $T_{i}$, for some $i$. Now $T_{i}-x-y$ must consist of exactly three components $T_{1}, T_{2}$, and $T_{3}$, where, say, $x$ joins $T_{1}$ and $T_{2}$ and $y$ joins $T_{2}$ and $T_{3}$. For $k=1,2$, and 3 , let $G_{k}$ denote the maximal connected subgraph of $G-x-y$ containing $T_{k}$, respectively. By definition, the $G_{k}$ 's are either identical or point-disjoint.

Suppose $G_{2} \neq G_{3}$. Then $G_{2}$ and $G_{3}$ must be point-disjoint and thus $y$ is a bridge of $G$, contradicting the assumption that $G$ is a block. Thus $G_{2}=G_{3}$. Similarly, $G_{1}=G_{2}$. Thus $G_{1}=G_{2}=G_{3}$ and $G-x-y$ is connected, contrary to the definition of the set $\{x, y\}$. This completes the proof.

We next establish upper and lower bounds for the number of lines in any b.l.c. graph in terms of the number of points.

THEOREM 10. If $G$ is a b.l.c. graph with $|V(G)|>3$, and if $L$ denotes the number of lines in $G$, then $|V(G)| \leqq L \leqq 2|V(G)|-4$, and these bounds are best possible.

Proof. The lower bound is clear, since $G$ is in particular a block. If $G$ is a cycle, then the lower bound for $L$ is assumed.

If $G$ is not a cycle, $G$ must contain at least two points of degree greater than two. Let $S$, as before, denote the set of points of degree two, and let $k$ be the number of components $T_{i}$ of $G-S$. Then $2 \leqq|S| \leqq|V(G)|-2$ and $2 \leqq k \leqq|S|$. Thus we have

$$
\begin{aligned}
L \leqq 2|S|+\sum_{i=1}^{k}\left(\left|T_{i}\right|-1\right) & =2|S|+\left(\sum_{i=1}^{k}\left|T_{i}\right|\right)-k \\
& =2|S|+|V(G)|-|S|-k \\
& =|S|+|V(G)|-k \leqq|S|+|V(G)|-2 \\
& \leqq|V(G)|-2+|V(G)|-2 \\
& =2|V(G)|-4 .
\end{aligned}
$$


To show that this upper bound is best possible, we note that if $G=$ $K(2,|V(G)|-2)$, the complete bipartite graph with two points of one color and $|V(G)|-2$ points of another color, then $G$ is a b.l.c. graph with exactly $2|V(G)|-4$ lines.

Our final task is to determine which b.l.c. graphs are planar. To treat this problem we introduce some terminology. Let $G$ be any graph. Denote by $G_{0}$ the graph obtained from $G$ by replacing every path in which each intermediate point has degree two by a single line. $G_{0}$ is then said to be the contraction of $G$. The reader will recall that Kuratowski's Theorem states that a graph $G$ is planar if and only if it contains no subgraph which can be contracted to $K_{5}$ or to $K(3,3)$.

THEOREM 11. A b.l.c. graph $G$ is planar if and only if its tree-condensation graph $G^{*}$ is planar.

Proof. Clearly if $G$ is planar, then so is $G^{*}$. To prove the converse, assume $G$ to be nonplanar. Hence $G$ contains a subgraph $H$ which can be contracted to $K_{5}$ or to $K(3,3)$. Suppose first that $K_{5}$ is the contraction of $H$. We shall call those points of $H$ which remain points in $K_{5}$ principal. Since the degree in $G$ of each principal point of $H$ is three, each principal point must lie in a component tree of $G-S$.

We proceed to show that no component tree of $G-S$ may contain more than one principal point. Let $T_{0}$ be such a component tree and let the principal points of $H$ be $u_{1}, u_{2}, u_{3}, u_{4}$, and $u_{5}$. Further, let $P_{i j}$ denote the path in $H$ joining $u_{i}$ and $u_{j}$ and having all intermediate points of degree two. Suppose $T_{0}$ contains more than one of the $u_{i}$. To be precise, there are four possible cases:

(1) Suppose $T_{0}$ contains exactly two principal points, say $u_{1}$ and $u_{2}$. Then $C=P_{13}+P_{32}+P_{24}+P_{45}+P_{51}$ is a cycle in $G$ and $C \cap T_{0}$ is nonempty and disconnected, contradicting Theorem 4.

(2) Suppose $T_{0}$ contains exactly three principal points, say $u_{1}, u_{2}$, and $u_{3}$. Then the cycle $C=P_{14}+P_{42}+P_{23}+P_{35}+P_{51}$ and $T_{0}$ contradict Theorem 4 .

(3) Suppose $T_{0}$ contains exactly four principal points, say, $u_{1}, u_{2}, u_{3}$, and $u_{4}$. Further suppose that $P_{12}, P_{23}$, and $P_{34}$ all lie in $T_{0}$. Then $P_{24}$ does not lie entirely in $T_{0}$, for if it did, $T_{0}$ would contain the cycle $P_{23}+P_{34}+P_{24}$. Thus the cycle $C=P_{12}$ $+P_{24}+P_{45}+P_{51}$ and $T_{0}$ violate Theorem 4. Hence one of $P_{12}, P_{23}$, and $P_{34}$ fails to be entirely within $T_{0}$. Then the cycle $C=P_{12}+P_{23}+P_{34}+P_{45}+P_{51}$ and $T_{0}$ contradict Theorem 4.

(4) Suppose all principal points of $H$ lie in $T_{0}$. Then one of $P_{12}, P_{23}, P_{34}, P_{45}$, and $P_{51}$ must not lie in $T_{0}$, say $P_{12}$. But now if any other of these five paths also fails to lie in $T_{0}, C=P_{12}+P_{23}+P_{34}+P_{45}+P_{51}$ and $T_{0}$ violate Theorem 4 . Thus assume that each of the five paths, other than $P_{12}$, lies in $T_{0}$. Then in particular, $P_{24}$ cannot lie in $T_{0}$, so that the cycle $C=P_{12}+P_{24}+P_{45}+P_{51}$ and $T_{0}$ contradict Theorem 4 .

Since all four cases lead to contradictions, the proof that each principal point of $H$ lies in a different component tree of $G-S$ is complete.

Next assume that there is a component tree $T_{0}$ of $G-S$ such that $P_{i j} \cap T_{0} \neq \varnothing$ and 
$P_{k m} \cap T_{0} \neq \varnothing$, where $P_{i j}$ and $P_{k m}$ are different. Then clearly $P_{i j}$ and $P_{k m}$ must have a common endpoint, for if not, then $C=P_{i j}+P_{j m}+P_{m k}+P_{k i}$ is a cycle such that $C \cap T_{0}$ is nonempty and disconnected, again contradicting Theorem 4. Suppose that $u_{i}$ is this common endpoint; i.e., suppose that $u_{i}=u_{k}$. Then $u_{i} \in V\left(T_{0}\right)$, for if not, then the cycle $C=P_{i j}+P_{j m}+P_{m i}$ and $T_{0}$ contradict Theorem 4 .

The above results suffice to show that if $G$ contains a subgraph which may be contracted to $K_{5}$, then so does $G^{*}$. A similar result holds for subgraphs contractible to $K(3,3)$. The details are left to the reader. We have thus shown that if there is a subgraph of $G$ contractible to $K_{5}$ or to $K(3,3)$, then there is a subgraph of $G^{*}$ with this same property. The proof of the theorem is thus complete.

The preceding theorem is in a sense the best result possible concerning the planarity of b.l.c. graphs. To realize this, one need only note that the next obvious question is: "What graphs can occur as tree-condensation graphs of b.l.c. graphs?" The answer is that any block $B_{0}$ can be thought of as the tree-condensation graph of some b.l.c. graph $B$. To find one such $B$, just insert one new point on each line of $B_{0}$. The resulting graph $B$ then has the property that every line is incident with a point of degree two. Such a graph is clearly block-line-critical.

Added in proof. The author wishes to acknowledge the appearance of a paper on this subject by G. A. Dirac (Minimally 2-connected graphs, J. Reine Angew. Math. 228 (1967), 204-216) while our paper was in press. In particular, he obtains a structural result similar to our Theorem 2 .

\section{REFERENCES}

1. T. Gallai, Uber extreme Punkt- und Kantenmengen, Ann. Univ. Sci. Budapest 2 (1959), 133-138.

2. O. Ore, Theory of graphs, Amer. Math. Soc. Colloq. Publ., Vol. 38, Amer. Math. Soc., Providence, R. I., 1962, p. 225.

YALE UNIVERSITY,

New Haven, Connecticut 